\title{
Expedition to Mars North Pole and Creation: There Is a Scientific Research Base
}

\author{
Alexander Rubinraut \\ Design Office "Expplanet”, Munich, Germany \\ Email: rubinraut32@mail.ru
}

How to cite this paper: Rubinraut, A. (2020) Expedition to Mars North Pole and Creation: There Is a Scientific Research Base. Advances in Aerospace Science and Technology, 5, 20-43.

https://doi.org/10.4236/aast.2020.51002

Received: January 15, 2020

Accepted: March 9, 2020

Published: March 12, 2020

Copyright (c) 2020 by author(s) and Scientific Research Publishing Inc. This work is licensed under the Creative Commons Attribution International License (CC BY 4.0).

http://creativecommons.org/licenses/by/4.0/

(c) (i) Open Access

\begin{abstract}
A project of the expedition to the north pole of Mars, which is carried out in two stages, has been developed. In the first stage, a space refueller and a mobile solar battery are delivered to the icy surface of the north pole of Mars. The delivery is carried out with the help of electric rocket ER-7, driven by the electric rocket engine "MARS", and the source of electricity is a solar battery from gallium arsenide. In the second stage, the space train is formed from two interconnected ER-7 rockets and an orbital takeoff-landing capsule TLC-2, in which three astronauts land on the surface and carry out a complex of physical, biological and geological researches on Mars surface. The space refueller has been developed, which melts the water ice on the surface of Mars and with the help of an electrolyser and a liquefier produces fuel and oxidizer for the operation of the chemical rocket engine of the takeoff-landing capsule. To move astronauts from the north pole to the equator, a spacecraftmarsoplane, which consists of the electric rocket ER-7 and the takeoff-landing capsule TLC-1 is developed. The duration of the expedition is 110 days at a cost of 2 billion dollars.
\end{abstract}

\section{Keywords}

Mars North Pole, Electrical Rocket ER-7, Takeoff-Landing Capsule TLC-1 and TLC-2, Space Refueller, Marsoplane, Superconducting Electrorocket Engine MARS

\section{Introduction}

January 2019 has marked the 15th anniversary of George W. Bush's proclamation of the 21st century American space program.

The program had provided resume regular lunar missions in 2014 and implementation of the first mission on Mars in 2018. 
The flight to Mars on the NASA spacecraft had provided for separate launch of the spaceship and the space capsule "Orion", in which the crew is delivered into Earth orbit.

The detailed description of the Martian expedition is given in [1] and [2].

Unfortunately, it should be stated that the works on this project in 2011 were closed.

But now we are witnessing an NASA attempt to revisit the Mars problem, the project description of the new NASA program is given in [3] and [4].

The project is based on the creation of super-heavy carrier rockets SLS with a payload capacity of up to 130 tons.

Constructively, this rocket resembles rocket "Saturn", but around the hull with oxygen-hydrogen rocket engines two additional solid-state boosters are installed (like in "Ariane" rocket). The project involves the installation of two different SLS head parts. The first head part is a spaceship for flight of an expedition of 6 people from Earth orbit to Mars orbit.

The second head part of the SLS rocket is the Orion space capsule, which is equipped with a rescue rocket, as it was in the design of the Saturn rocket.

The spacecraft for interorbital flight consists of two cylinders and a cone with a diameter of 5 to 12 meters with a total length of 24 meters.

In the head part, there is a residential module, on the cylindrical surface of which is unit for docking with the capsule "Orion". The residential module is connected along axis to tanks-containers filled with fuel and oxidizer which are stored in liquid state and are intended. For the operation of a chemical rocket engine, four RS/25D/E chemical rocket engines are located in the tail section. The project involves reaching the orbit of Mars 11 months after launch. After that, it is planned to fly around the planet Mars and to land on its surface.

Currently, it is not clear how the astronauts will perform landing on Mars surface, takeoff from the surface and return inside the spacecraft. Probably for these operations the Orion capsule can be used. After the Mars flyby, the spacecraft returns into Earth orbit.

On the 540th day of flight, the crew, after docking, moves into the Orion capsule, which was previously delivered by the SLS rocket into Earth orbit and which then is splashed down in the Pacific Ocean. The published data shows that NASA engineers are still far from solving the problem. In addition, it is obvious that due to the need to build new carrier rockets, the cost of the project will be very high.

It should be noted that the accepted design of the spacecraft does not provide for the creation of artificial gravity in the cabin of astronauts during the orbital flight.

Who can now vouch that in the human organism as a result of a long stay in weightlessness will not occur such changes that astronauts who have been on expeditions, will no longer be able to live on Earth.

These serious flaws of the new NASA project show that it is based on technical solutions of 50 years ago, does not permit to solve the assigned task. 
At the same time, studies carried out in recent years by the author of this article have shown that for the successful implementation of the expedition to Mars it is necessary to go another, alternative way.

The task can be solved if the orbital flight is carried out with the help of an electric rocket equipped with an electro rocket engine, which has a specific momentum of many times greater than that of a chemical rocket engine.

This property is acquired by an electric rocket thanks to a new constructive solution, which is realized by the usage of high-temperature superconductors developed by physicists in the 21 st century.

The first step on the way of implementation of flights towards the planets of the solar system was the invention in 2006 of the superconductor electric rocket engine MARS of the magnetoplasma type. The new engine design provides for the installation of a superconducting magnetic system in which the current is directed parallel to the longitudinal axis of the engine. This allows you to increase the efficiency of the engine from $60 \%$ to $94 \%$ [5].

The second step was taken by the author in 2016, when the design of the electric rocket ER-7 was developed, capable of transferring a payload of up to 10 tons from Earth orbit to Mars orbit the electric rocket ER-7 was named after the historic R-7 rocket, which was created by S. P. Korolev. The rocket ER-7 (which design is described in [6]) is located in the assembled form, in the head part of the carrier rocket the "Delta 4 Heavy", serially produced by Boing (USA).

The carrier rocket "Delta 4 Heavy" is equipped with three RS-68 chemical engines, which ensure putting of 26 tons of payload into orbit around the Earth.

The fulfilled design developments show that the interorbital rocket ER-7 can become a modular base for the formation of space trains to the planets Mars, Venus, Mercury and Ceres.

In this paper, the project of the expedition to the north pole of Mars is considered. We are witnessing an activation in the process of studying Mars, which has been held for several years. Spacecrafts which are regularly sent from Earth, bring new scientific results.

The question of the presence of water on the surface of Mars is particularly interesting.

The answer to this question was received, when NASA's Phoenix probe has landed on the north pole of Mars in 2008 and has received water out Martian ground [7].

Owing to this circumstance, it is possible to refuel tanks with fuel and oxidizer right on the surface of Mars, while using water, which in the form of ice is in the polar cap of Mars, as the source material for obtaining the necessary components-hydrogen and oxygen. The similar form of water ice usage was previously developed by the author for an expedition to the planet Jupiter [8]. Hydrogen and oxygen obtained from water ice can be used not only to refuel chemical rocket engines, but also to refuel the fuel cells of the Mars rover and for the expedition's own needs. 
Another important circumstance that determined the landing site of the expedition at the north pole of Mars is related to the purpose of the expedition. The most important scientific task of the expedition is to find the simplest living organisms on the surface of Mars. And to look for them first of all one need where there is water, that is on the icy surface.

During the development of the expedition project, a study was carried out, which showed the possibility and expediency of using solar panels from arsenide-gallium as a source of energy for electric rocket.

At the same time, it should be constantly taken into account that due to the increase in the distance to the Sun during the movement of the rocket, the power of the solar panel is continuously reduced.

\section{The Concept of Carrying Out of an Expedition on the North Pole of the Planet Mars}

Calculations and design studies conducted with the help the mathematical model showed that the expedition to the north pole of Mars is advisable to conduct with the help of space trains, which are assembled in the orbit of the Earth out of electric rockets and takeoff-landing modules. The number of electric rockets and modules in each train depends on its purpose.

Each component of the rocket space train is put into circumterrestrial orbit with the help of "Delta 4 Heavy" carrier rocket. Interorbital flight of the space train is carried out by a rocket, which is moved by an electric rocket engine MARS of new design developed and researched by the author [5] [6]. For power supply of the electric engines on the electric rocket an collapsible solar battery based on arsenide-gallium is installed. The working substance for the operation of electric motors is placed in a cryogenic tank located along horizontal axis.

The take-off-landing modules of various purposes are equipped with a chemical hydrogen-oxygen engine, which allows the module to move along orbit around Earth and around Mars.

The fulfilled studies have shown that an expedition to the north pole of Mars is advisable to conduct in two stages. In the first stage, into orbit passing through Mars north pole, the working substance for return flight of the expedition to Earth, is delivered.

The scheme of the cargo interorbital space train for the first stage is shown in Figure 1.

The flight is carried out with the help of an electric rocket ER-7 (5), along axis of which a tank with liquid nitrogen (3) is located. The rocket is moving along interorbital trajectory using 4 electric rocket engines MARS (6). Electric rocket engines are powered by solar panels (2). With the help of the space train, shown in Figure 1, refueller 7 and solar battery 8 are delivered into Mars orbit.

After assembling space train (Figure 1) in Earth orbit in automatic mode with the help of docking units 4 and 5 begins the movement of the space train (Figure 1) along the calculated trajectory from Earth orbit to Mars orbit. The train reaches the planet Mars and enters the Mars orbit at a distance of $200 \mathrm{~km}$ from 


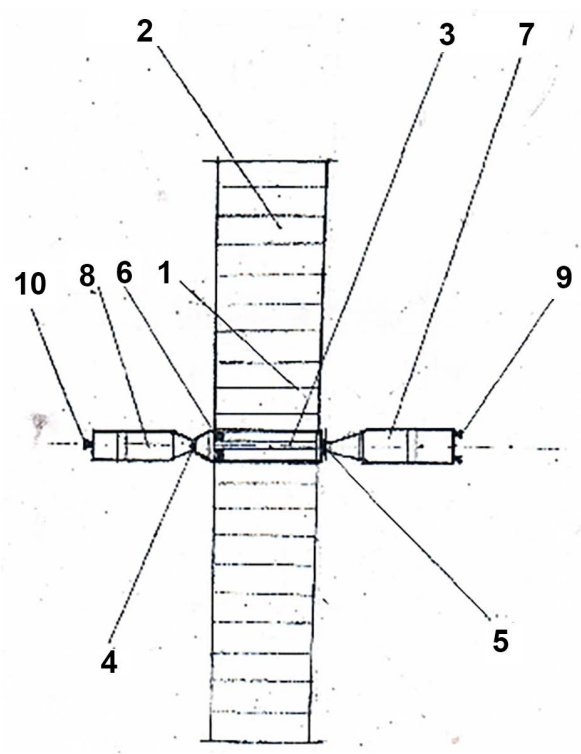

Figure 1. Cargo space train.

its surface. The calculations showed that with the optimal mode of movement of the rocket train (Figure 1) it should be accelerated to a speed of $35 \mathrm{~km} / \mathrm{s}$ within 6 days, and then must continue the flight with the electric rocket engines turned off during 166 days. At the last stage of the flight, the electric rocket engine should be switched on in braking mode and within 6 days to slow down the space train up to a speed of $5 \mathrm{~km} / \mathrm{s}$. At that time, the weight of the working substance-nitrogen, which is in tank 3 , is decreased from 14 to 11.5 tons. In the future, this reserve of the working substance will be used for the return flight of the expedition into the Earth's orbit.

After entering Mars orbit, the space train (Figure 1) is devided with the help of docking units 4 and 5. According to the plan of the first stage of the expedition, space refueller 7 is landed at the intended point. Its coordinates are: 85 degrees north longitude and 330 degrees north latitude of Mars on its icy surface. The chemical rocket engines 9 are turned on and the refueller loses speed. When it enters the Martian atmosphere, the parachute landing system automatically turns on (at altitude of $30 \mathrm{~km}$ ).

After landing of the refueller 7, in the similar way, the landing on the surface of Mars the rocket 8 in which is there a solar battery (in the collected form) is carried out. The landing process is performed in the following way: the chemical rocket engine 10 is turning on and the rocket 8 departs from the electric rocket ER-7. After losing speed, the rocket 8 is moving under the force of gravity towards the surface of Mars. When entering the Martian atmosphere, a parachute system is activated and a soft landing of the rocket 8 on the surface of Mars is carried out in close proximity to the space refueller landing site.

The ER-7 rocket remains in orbit around Mars and is used as a satellite system for the Mars surface observation.

During the last stage of the expedition, the ER-7 electric rocket will be in- 
cluded in rocket complex of return flight, i.e. for the flight from Mars orbit to Earth orbit.

The formation diagram of the rocket complex for the expedition crew flight from Earth orbit to the orbit of Mars is shown in Figure 2.

Let's see how the process of assembling the space train (Figure 2) in the Earth's orbit takes place. Such space train can be called cargo-passenger. It is assembled by docking along axis of two electric rockets ER-7. Electric rocket 1 is launched into Earth orbit in assembled form being in the head part of the carrier rocket "Delta 4 Heavy".

After unfolding of the solar panels, rocket 1 docks with the takeoff-landing capsule 6 (TLC-1). The takeoff-landing capsule 6 (TLC-1) is delivered into orbit around the Earth also using "Delta 4 Heavy" carrier rocket. After docking, we get an autonomous spacecraft, with which the crew can fly anywhere on the surface planet Mars. In the future, the complex consisting of rocket 1 and TLC-1 (6), we will call "Marsoplane".

The second half of the space train (Figure 2) is formed by electric rocket ER-7 (2), which is put into orbit around the Earth by the carrier rocket "Delta 4 Heavy".

After unfolding of the solar panels, of the rocket 2, the docking of the rocket with the takeoff-landing capsule TLC-2 (3) is carried out. It is in the capsule TLC-2 that the crew of the expedition consisting of three astronauts is located. But at first, it is necessary to deliver astronauts to ISS. For this purpose, one can use the known systems of delivery of astronauts to ISS: "Soyuz", "Orion" or "Dracon". Therefore astronauts expect the beginning of the interorbital flight being at ISS.

The takeoff-landing capsule TLC-2 is launched into Earth orbit using the carrier rocket "Delta 4 Heavy". After launch, it goes into rapprochement with ISS and is mooring to it. For this purpose, chemical rocket engine 9 is used. With the help of docking unit 10 located in the nasal part of TLC-2 the connection of TLC-2 with a ISS moorage takes place.

Astronauts leave ISS and pass into the cabin of TLC-2. The takeoff-landing capsule 3 departs from ISS and using chemical rocket engine 9 approaches electric rocket 2.

With the help of the second docking unit 8 , which is located along the transversal axis of the capsule, docking of rocket 2 and capsule 3 is carried out.

The final manoeuvre in Earth orbit before the start of the orbital flight is the connection of rocket 1 and rocket 2 . During this manoeuvre, the rocket convergence is carried out with the help of electric rocket engines 4 , and the docking-with the help of docking unit 7.

The electric rocket train, shown in Figure 2, is ready to fly from Earth orbit to Mars orbit.

It remains only to turn on the artificial gravity system, which arises in the cabin of TLC-2 (3) when it is rotating around the transversal axis with the help of superconducting bearing 8 . 


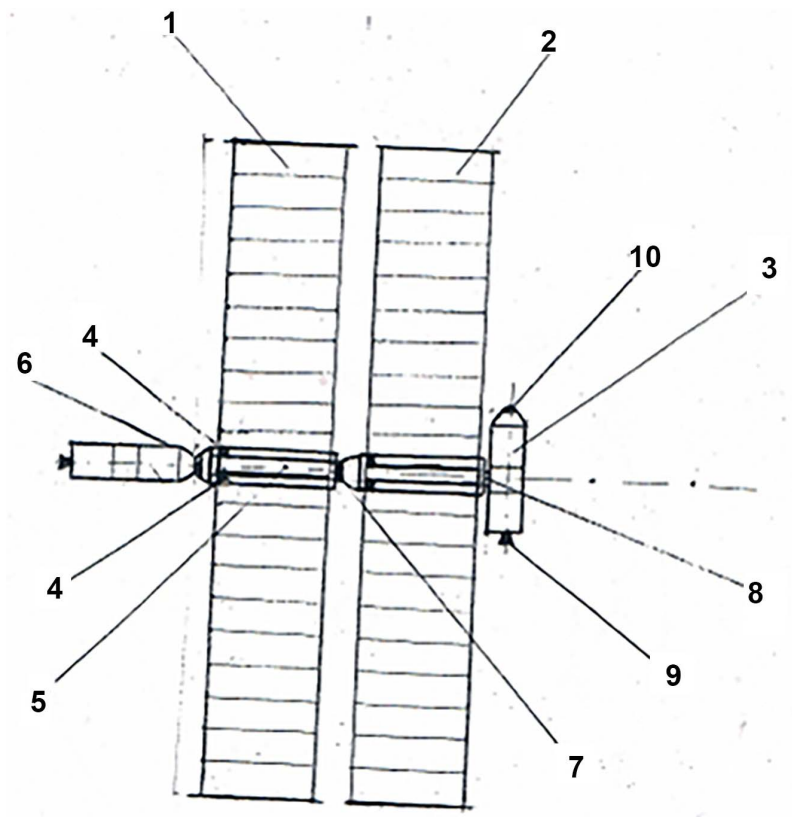

Figure 2. Cargo-and-passenger space train.

Astronaut-driver turns on electric rocket engines 4, and the space train begins to increase the speed of movement. Having gained the second space speed, the space train (Figure 2) goes into the calculated trajectory of flight.

The flight trajectory is built using an astrodynamical program that continuously determines the forces of interaction between the space train and the Sun, Earth, its satellite-Moon and the planets Venus and Mars at their simultaneous movement along orbits [9].

In so doing the picture of the gravitational field of the solar system in the area of motion of the space train, which is the body of variable mass, which is under the influence of this field, is determined.

The calculation program provides changing the thrust of rocket engines depending on the distance to the Sun. This factor is taken into account at each point of the trajectory using the method of sequential approximations.

The resulting flight trajectory is shown in Figure 3, and the process of changing the speed and mass of the train as well of the power of the on-board energy source in time is shown in Figure 4.

As it can be seen from Figure 3, the start of the space train (Figure 2) begins at the moment when the Earth is at the point O. Mars at this time is at the point O', and while moving along its orbit, it makes one turn around the Sun in 676 Earth days.

As it can be seen from Figure 4, the acceleration of the space train to speed of $100 \mathrm{~km} / \mathrm{s}$ takes place within 19 days. At this time, the electric rocket engines operate at maximum capacity, which magnitude is continuously decreasing. After a three-day inertia flight, the rocket makes a 180-degree u-turn. After that, during 18 days the space train reduces the speed of movement from $100 \mathrm{~km} / \mathrm{s}$ to 3.6 $\mathrm{km} / \mathrm{c}$. At this speed, the space train becomes a satellite of Mars. Thus, the flight 


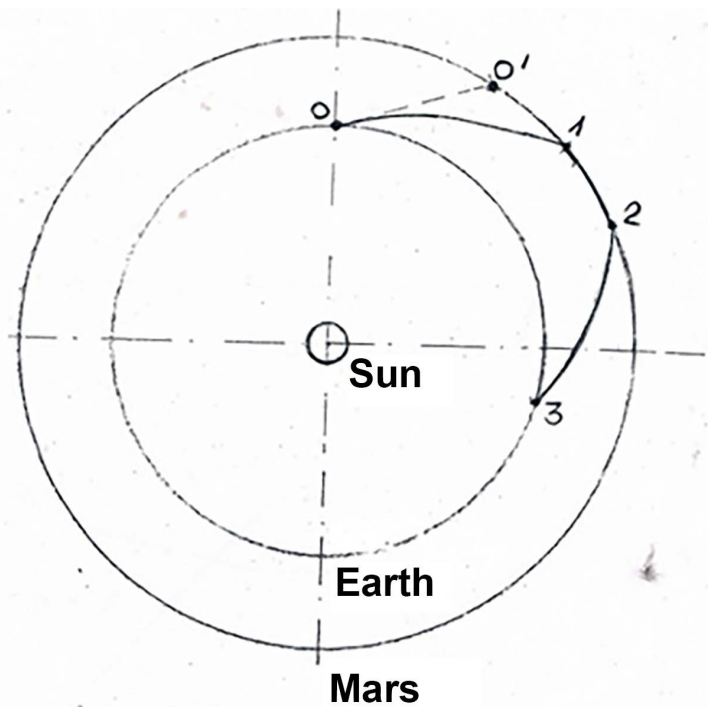

Figure 3. Space train flight trajectlory.

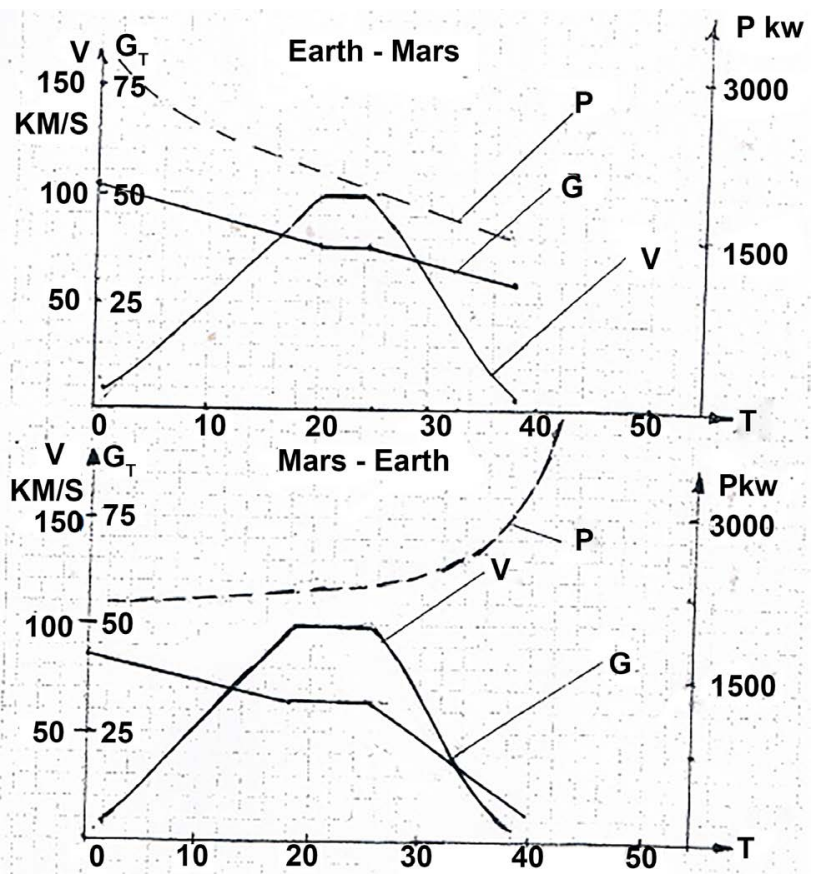

Figure 4. Space train flight characteristics.

from Earth orbit to the orbit of Mars takes 40 days. According to the expedition program, astronauts conduct scientific observations.

Using telescopes, they observe Mars satellites-Phobos and Deimos. Astronauts are also monitoring the movement of a space train that was launched into Mars orbit earlier and has delivered a refueller and a solar battery on the surface of Mars (Figure 1).

The space train (Figure 2) is divided by means of the docking unit 7 . The rocket engine of the electric rocket ER-7 (1) is switched on and the rocket ER-7 (1) departs from the electric rocket ER-7 (2). With the help of docking unit 8 the 
TLC-2 (3) is disconnected from the rocket ER-7 (2). Astronaut-driver switches on chemical rocket engine 9. TLC-2 slows down and moves away from electric rocket 2, which remains in orbit of Mars.

At achievement of speed of $3.5 \mathrm{~km} / \mathrm{s}$, the TLC-2 (3) rushes towards the surface of Mars with acceleration of $0.38 \mathrm{~g}$. When entering the atmosphere of Mars at a distance of $60 \mathrm{~km}$ from its surface, the parachute system is triggered. TLC (3) is approaching the surface of Mars. With the help of a chemical rocket engine 9, the astronaut pilot makes a smooth landing of the capsule 3 in the point on the icy surface of Mars with coordinates $85^{\circ} 330^{\circ}$, where two spacecraft-space refueller and a solar battery-are waiting for the expedition arrive.

After landing on the surface of Mars, the astronauts leave the landing capsule TLP-2 and begin to work according to the expedition plan. First, it is necessary to activate the refueller and bring it into a state of readiness for operation. Secondly, it is necessary to provide all the devices with power. Using a special device, the astronauts install 2 coils on which there are photovoltaic converters of electric power. Then the astronauts deploy on the surface two solar panels each with a capacity of $180 \mathrm{~kW}$. The first battery is connected to the ice melter which is in the refueller. The second battery is connected to a system of electrolyser and liquefier.

From the cargo compartment of the solar panel, the astronauts unload a vehicle to move on the icy surface of Mars and the prefabricated inflatable residential complex "Iglus", which was designed by NASA specifically for Martian expeditions [10].

Once the solar panel is connected to the refueller, the astronauts turn on all the provision systems. They control the process of refuelling with the working substance, the formation of pool with unfrozen water and control the work of the electrolyser and liquefier.

To carry out the work of assembling and adjustment of the refueller, the astronauts connect a new device-a robot-astronaut [11]. Astronaut Robonaut-2, who underwent special training on the ISS back in 2014, begins servicing of the space refueller.

At first, with the help of the refueller, the tanks of the takeoff-landing capsule TLC- 2 , in the cabin of which the astronauts are staying, are being filled with liquid hydrogen and liquid oxygen. Now TLC- 2 is permanently on standby readiness to return to Earth orbit.

Astronauts begin to perform scientific research. The research program involves primarily the study of the ice cover of the north pole of Mars. It is supposed to make another attempt to search for life on the ice of Mars. For this purpose, with the help of a tracked rover, two astronauts, one of them a professional biologist, make a travel along icy surface. The route of the travel is shown in Figure 5.

Currently, a number of firms are working on creation of a Mars rover. This project gave preference to the rover, which was developed by engineers at Londoner Royal College, the description of which is given in [12]. 


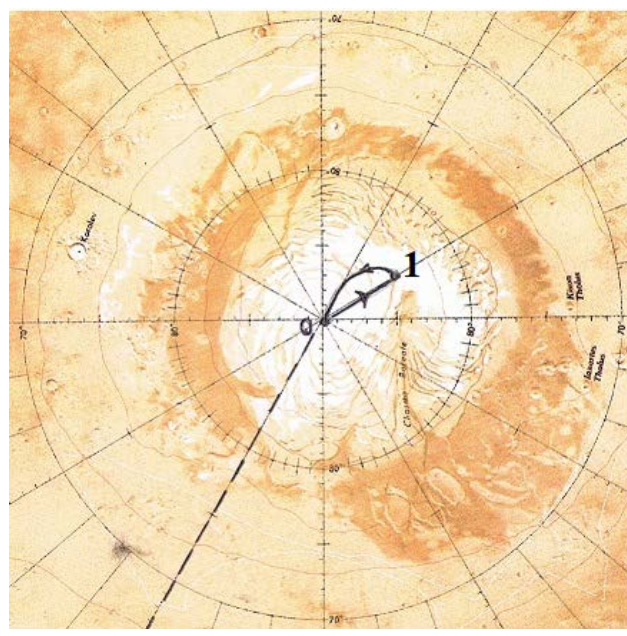

Figure 5. Expedition route at north pole region (at physical map of Mars [13]).

As it can be seen in Figure 5, the rover begins its movement along route from the point $1\left(85^{\circ} / 330^{\circ}\right.$, expedition base) towards the North Pole. Astronauts move on the icy surface at a speed of $20 \mathrm{~km} / \mathrm{h}$.

At every stop, ice is drilled and its composition is analysed.

Passing $100-120 \mathrm{~km}$ per day, the astronauts reach the point 0 (North Pole) with coordinates $\left(0^{\circ} / 0^{\circ}\right)$ and in 5 days return to the base.

The expedition program also provides for research on a stretch of the surface of Mars close to the equator.

As it was indicated earlier, to move astronauts from the base at the north pole of Mars to the intended points on its surface in the project developed a new spacecraft—a marsoplane, shown in Figure 6.

\section{Continuation of the Expedition-Equatorial Flight on the Marsoplane (Figure 6)}

The marsoplane consists of space locomotive 1 and takeoff-landing capsule TLC-1 (2), which is docked along axis of the rocket ER-7 and is its payload.

The takeoff-landing capsule TLC-1 is delivered to the Mars surface during a flight from Earth orbit to Mars orbit. In Figure 2 it is designated by index 6 .

After separation with the help of docking unit 3 the takeoff-landing capsule TLC-1 with the help of chemical rocket engine 4 smoothly descends on the surface of Mars in close proximity to the refueller.

With the help of a refueller, the astronauts are filling the tanks with fuel and oxidizer providing operation of the chemical rocket engine 4.

The TLC-1 design provides for operation of the chemical engine when taking off from the surface at the north pole and when entering into orbit around Mars. After docking capsule 2 and locomotive 1, the marsoplane in the assembled form (Figure 6) makes one turn around Mars at an altitude of $250 \mathrm{~km}$ for 35 minutes.

The marsoplane is operated by an astronaut-pilot, which switches on electric rocket engines 5 and 6 . With the help of electric motors, the marsoplane is 


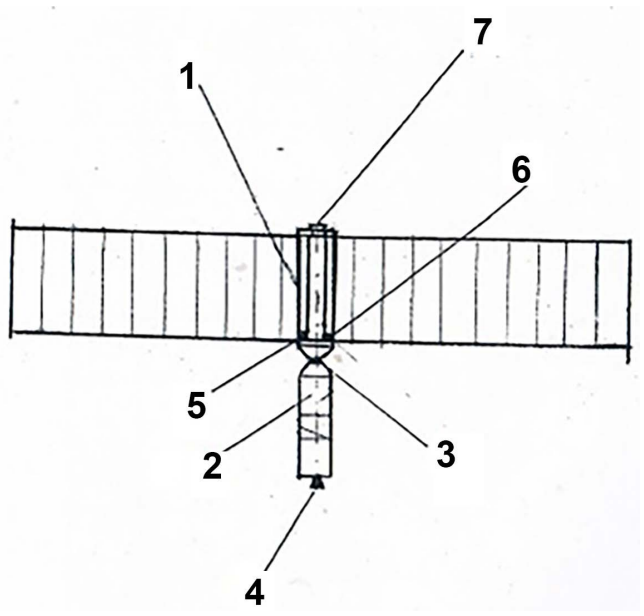

Figure 6. General view of marsoplane.

manoeuvring over the point of the north pole $0^{\circ} / 0^{\circ}$. As a result of the manoeuvre, the longitudinal axis of the marsoplane should be directed along the meridian, along which passes the trajectory of the flight to the chosen point on the surface of Mars.

After fixing the flight direction, the electric engines 5 and 6 marsoplane are being switched off, and the marsoplane begins to fly along the chosen orbit around Mars by inertia.

The first flight under the expedition program is carried out by the astronauts from the base at the north pole to the area of extinct volcanoes at the equator (to the point with coordinates of $15^{\circ} / 120^{\circ}$ ).

On the first leg of the flight, the marsoplane flies from point 1 (Figure 5) to point 0 (at the North Pole). Then the marsoplane makes a 30-degree turn and goes to the meridian $120^{\circ}$. Moving south, the marsoplane achieves point 2 at a latitude of $55^{\circ}$. The flight towards the equator continues from point 2 to point 3 (Figure 7).

The flight time along the orbit of Mars from point 1 to point 3 will be $10 \mathrm{mi}-$ nutes.

When approaching the landing point, the marsoplane flies past the highest peak of Mars-mount Olympus Mons, $27 \mathrm{~km}$ high.

Moving along meridian $120^{\circ}$, the marsoplane reaches the equator near the Ulysses Patera crater. The command is given for separation of the rocket ER-7 (1) and the takeoff-landing capsule TLC-1 this command is performed in automatic mode with the help of docking unit 3 . After the separation of the marsoplane the rocket continues to move around Mars along meridian $120^{\circ}$. The takeoff-landing capsule using a parachute lands at point 3, located at a distance of $200 \mathrm{~km}$ from the extinct Arsia Mons volcano. During the landing, the chemical rocket engine 4 works, providing a soft landing. At the landing place of TLC-1, the valley Marineris begins, which is stretched along the parallel $10^{\circ}$ and is a giant tectonic fault. 


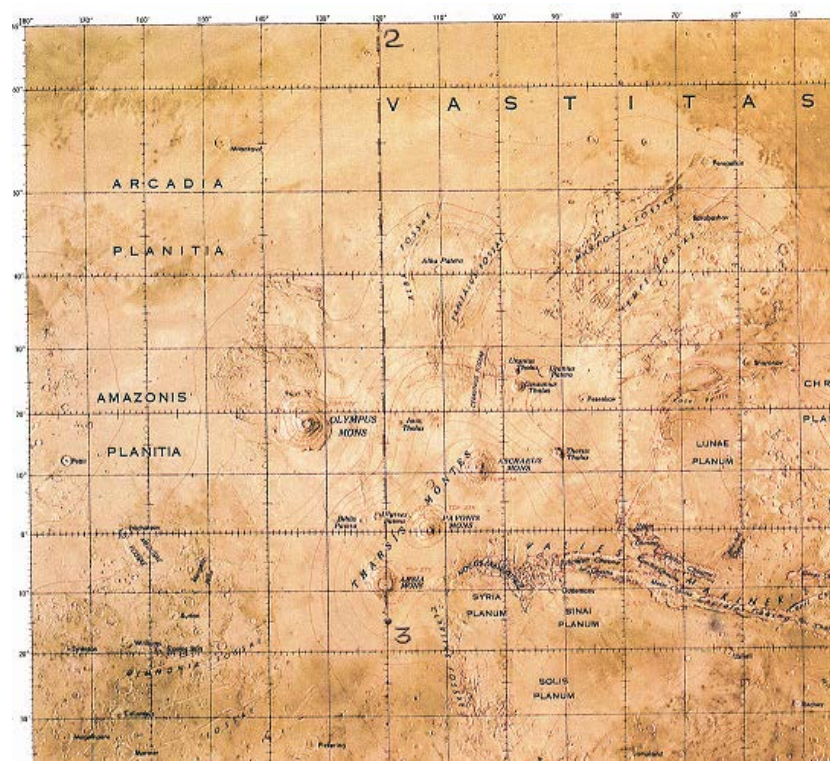

Figure 7. The route of expedition flight towards equator (at physical map of Mars [13]).

Astronauts begin to perform research on a special program: geology, astronomy, astrophysics, medical biology, etc. Along the surface of Mars, they move with the help of the rover of the prefabricated construction. For 10 days astronauts conduct a set of experiments using the latest instruments.

After the program's implementation and two-day rest, a work is carried out to prepare for the return flight to the base. It should be recalled that the crew must perform still another takeoff from the surface of Mars. Therefore the TLC tanks have fuel and oxidizer reserve for the operation of chemical rocket engine 4.

Astronauts take seats in the cabin of TLC-1. The astronaut-pilot switches on chemical rocket engine 4 (Figure 6). The takeoff-landing capsule takes off from the surface of Mars and in 190 seconds enters orbit around Mars. This orbit passes along meridian $120^{\circ}$ and it rotates the residual part of the marsoplane with electric motors and solar battery. After docking rocket 1 and takeoff-landing capsule 2 of the marsoplane using electric rocket engines 5 and 6 the correction of the direction to the north pole is being made.

The marsoplane is heading to the expedition base. Having made one turn around Mars, the marsoplane after 45 minutes of flight enters on the calculated point $\mathrm{O}$ (Figure 5) over the north pole of Mars.

The electric rocket engines 5 and 6 are switched on, the marsoplane makes $30^{\circ}$ turn and goes to the $330^{\circ}$ meridian. At the $85^{\circ}$ latitude astronauts perform separation of the marsoplane with the help of docking unit 3 (Figure 6).

Using a chemical rocket engine 4, the takeoff-landing capsule TLC-1 rushes towards the surface of Mars. At an altitude of $50 \mathrm{~km}$ above the surface of Mars, the capsule's parachute system is activated. With the help of chemical rocket engine 4, the capsule is softly landed at point 1 on the surface of Mars. While being at the base, the astronauts carry out work on the plan of the final stage of the 
expedition. According to this stage, astronauts must build a permanent laboratory at the north pole of Mars, which should carry out complex measurements of Mars nature parameters and transmit the information to Earth.

Such a laboratory can eventually be turned into a space station, where to conduct research with a constant change of composition of the expedition.

After the first expedition on the icy surface of the north pole of Mars remains a solar battery, as a permanent source of electricity. There remains also a space refueller, which is able to automatically produce oxygen and hydrogen from water ice and refuel the takeoff-landing capsules TLC-1 and TLC-2 by working substance for the operation of a chemical rocket engine.

The staying conditions of astronauts on Mars resemble those familiar to Earth's polar explorers. At the same time, it should take into account the climatic features of Mars.

As it already was pointed out, the expedition is provided with the installation of a special complex "Iglus" that protects astronauts from cosmic radiation and creates the necessary life comfort [5]. The complex has a system of constant supply of electricity, oxygen, hydrogen and water.

The maintenance of the complex for 18 months, when there will be no astronauts, is entrusted to the robot astronaut Robonaut-2.

The planned stay on Mars, which lasted 30 days, ends. The takeoff-landing capsule is put out of service (for 18 months) for use as part of a marsoplane during a re-expedition.

Astronauts take up places in the cabin of the takeoff-landing capsule TLC-2, the design description of which is given below.

For flight of the expedition from Mars orbit to Earth orbit in the orbit of Mars a rocket train, shown in Figure 8, is formed.

The rocket train is assembled on the orbit from three ER-7 rocket modules by manoeuvring with help of electric rocket engines 5 and 6 .

The rocket engine 7 of TLC-2 is switched on. The takeoff-landing capsule breaks off from the surface of Mars and in 180 seconds enters orbit around Mars, on which the space train moves (Figure 8).

After docking the takeoff-landing capsule and electric rocket train, the cruise electric rocket engine 6 of the rocket module 2 is switched on. The electric rocket train picks up speed, leaves Mars orbit and takes its course towards Earth orbit.

The trajectory of the electric rocket train is shown in Figure 3. At the beginning of the interorbital flight Mars is in its orbit around the Sun at point 2.

When moving, the space train must pass along the calculated trajectory from point 2 to point 3, where at the end of the flight. Will be located the planet Earth. The results of the calculation of the train parameters in time are shown in Figure 4.

Let's see how the train is moving. During the 18-day flight, the space train accelerates up to a speed of $100 \mathrm{~km} / \mathrm{s}$. This is due to the fact that when approaching the Sun the power of solar panels of three rockets 4,1 and 2 (Figure 8) continuously increases and after 20 days it becomes equal to $2.0 \mathrm{mw}$. 


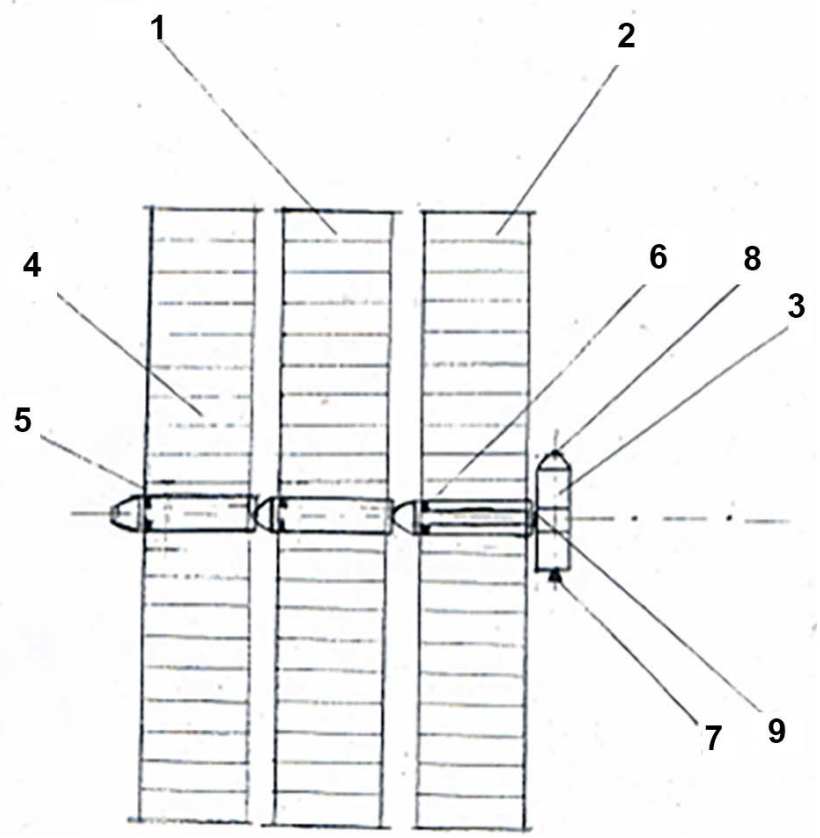

Figure 8. Space train for flight on the route Mars-Earth.

After 18 days, electric rocket engines 6 (Figure 8) are switched off, and the space train continues to move on inertia at a speed of $100 \mathrm{~km} / \mathrm{s}$ for 8 days.

After the manoeuvre of the space train, the direction of the thrust of the electric rocket engines changes by $180^{\circ}$. The braking of the space train starts and its speed within 8 days reduces to $10 \mathrm{~km} / \mathrm{s}$. Return flight from Mars orbit to Earth orbit takes 38 daysAfter entering the Earth's orbit, the space train is uncoupled (Figure 8).

Electric rockets ER-7-1,2,4 are sent to the orbital base, where they are refuelled with working substance-nitrogen. In the future, they are used for re-flight to Mars.

The takeoff-landing capsule TLC-2 with the crew on board with the help of chemical rocket engine 7 (Figure 8) is moving into orbit of the international space station ISS. The capsule steers to a ISS moorage and connects with it with the help of docking unit 8 . Through docking unit 8 the crew of the expedition moves from the capsule inside the ISS.

The expedition to the north pole of Mars, which lasted 110 days, ends. It should be taken into account that the Earth revolves around the Sun at an angular speed of 1.88 times greater than Mars. A second expedition to the north pole of Mars should be conducted at the moment of confrontation of the planets. And this will happen 22.5 months after the first expedition.

Regarding the cost of the expedition.

The preliminary flight will require three launches of the "Delta IV Heavy" carrier rocket.

The main flight will require four more launches of the "Delta IV Heavy" carrier rocket. 
Thus total cost of launches will be 600 million dollars.

The estimated calculated cost of the ER-7 rocket is 50 million dollars.

The estimated calculated cost of refueller 100 million dollars.

Cost of takeoff-landing capsule 80 million dollars.

The project estimated cost of the expedition is 2 billion dollars.

\section{The Electric Rocket ER-7}

The general view of the rocket is shown in Figure 9.

The ER-7 electric rocket is intended to create the thrust force of a space train using an on-board energy source and an engine. The payload, in this case, is the takeoff-landing capsule. The design of the case and its connection with the solar battery are shown in Figure 10.

The solar battery has a folding design. It is assembled from individual panels 4, which are made of carbon. From the outside in the panel, a layer of photovoltaic converter, which is made of arsenide gallium is being embedded. The panels are connected using hinges 16,17 . The cross-section of the case design is shown in Figure 11 and Figure 12.

Figure 11 shows the position when the solar panel is being already in assembled form while being delivered onto orbit around the Earth. From the outside, the structure is held by a cylindrical shell 4 . Installation of solar panels in working position is carried out with the help of sliding thrusts, which are fastened on longitudinal beams 5 and 6 .

In Figure 12, the fastening of the sliding thrusts 6 and 7 on the outer side of the end disk 2 is shown. Sliding thrusts are made in the form of concentric cylinders 6 and 7 inserted into each other.

The cylindrical case of the rocket 1 (Figure 10) made of aluminium alloy, serves as the basis of the design. End disks 2 and 10 are installed on both sides of the case 1.

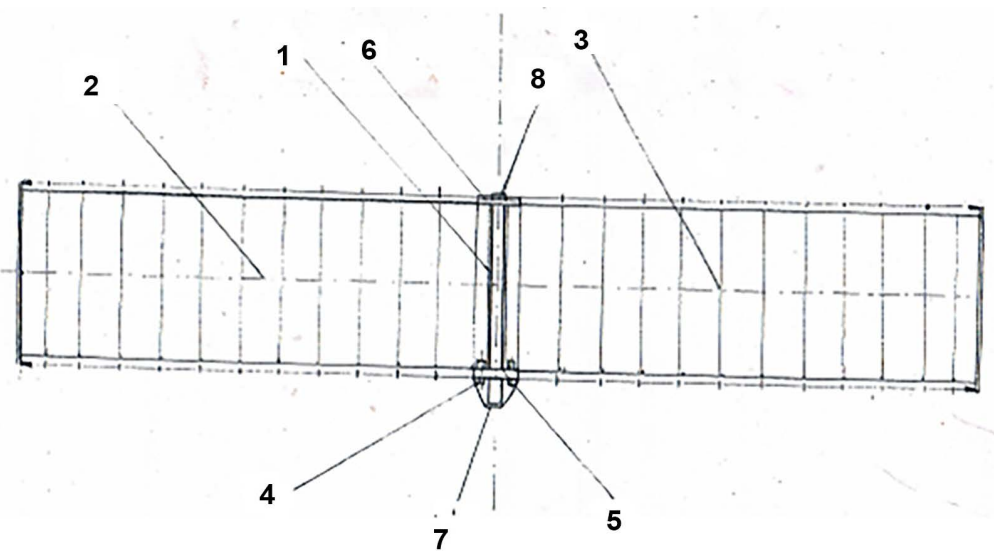

Figure 9. General view of ER-7 rocket. where: 1) body; 2) right solar battery; 3) left solar battery Solar batteries have panels consisting of cells made of gallium arsenide; 4) electrorocket engine MARS; 5) front end disk; 6) rear end disk; 7) front docking unit; 8) rear docking unit. 


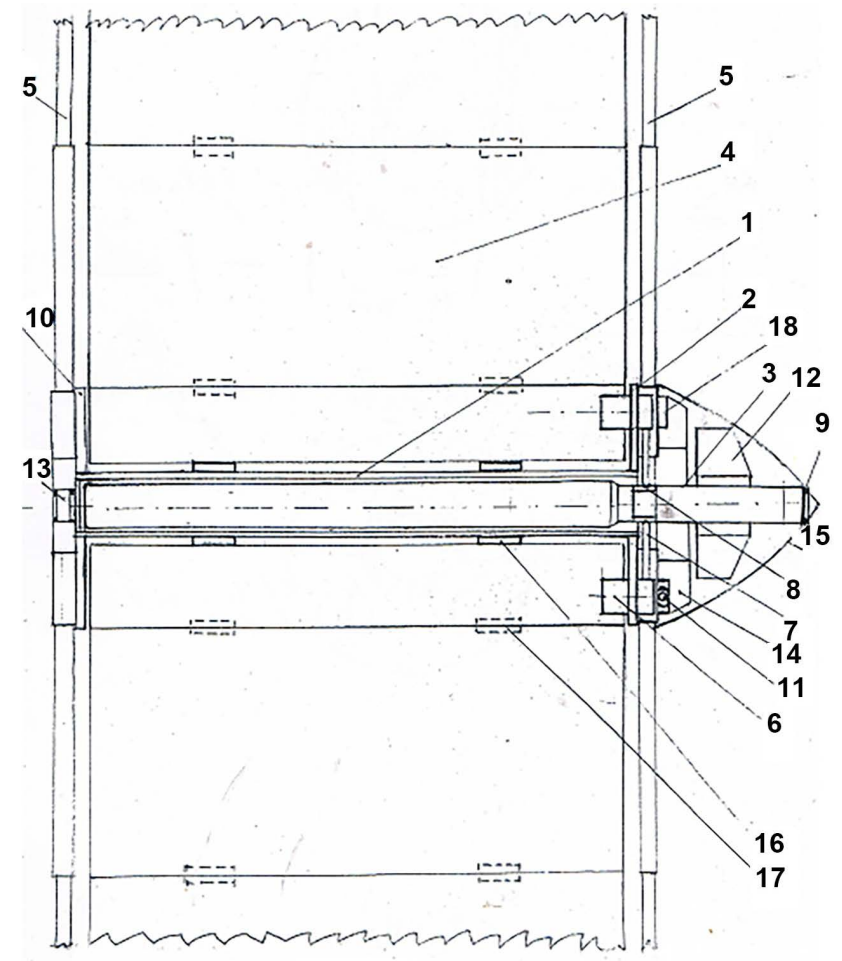

Figure 10. ER-7 rocket case (longitudinal section).

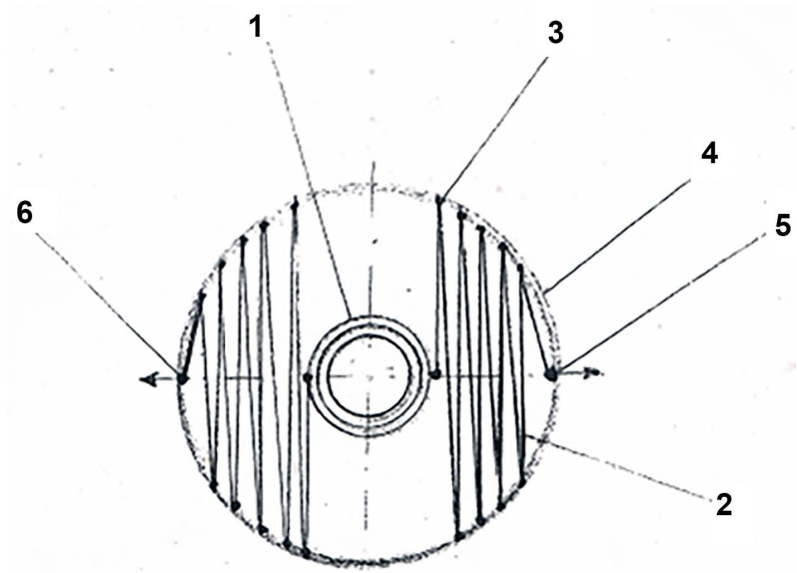

Figure 11. ER-7 rocket case (cross-section), solar battery installation.

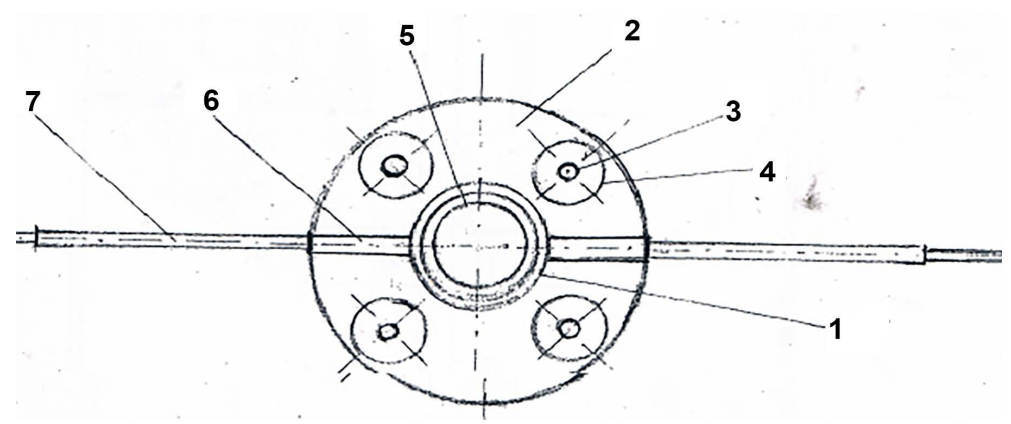

Figure 12. ER-7 rocket case (cross-section), installation of electric rocket engines. 
The tank 3, which houses the working substance of the electric rocket engine (nitrogen in liquid condition), is made of carbon. It has the form of a cryostat with screen-vacuum insulation. In the neck of cryostat 3 , the cryogenic pump 8 to pump liquid nitrogen is installed.

Electric rocket engines 6 are installed in the butt-end disk 2. To install electric rocket engines, the end disc 2 (Figure 12) has four holes 4 located at an angle of $90^{\circ}$.

In the nasal part of the rocket, there is an outer case 15, in the inner cavity of which the units of automatic control system 12 are installed.

\section{Superconductive Electric Rocket Engine MARS (Abbreviation of the German Name-Motor Für Die All Rakete Mit Der Supraleitung)}

The design of the superconductor electric rocket engine MARS is shown in Figure 13 (longitudinal section) and Figure 14 (cross-section).

The engine belongs to the class of magnetoplasm electric rocket engines with external exciting coil. Its main difference from the existing ones is that the coil 7 (Figure 13) excites in the engine's working chamber transversal magnetic field.

To do this, the current in the exciting coil 7 is directed along the longitudinal axis of the engine. The magnetic field, excited by coil 7 , is perpendicular to the electric current between the electrodes.

Another difference is that to increase the value of the transverse magnetic field, the coil is made of superconductor and is placed in cryostat 18 with liquid nitrogen (Figure 14).

The use of superconductivity also reduces power loss to minimum. The electric motor was patented by the author in Germany in 2013 [14]. In the working chamber along the axis of the engine is located cathode 1. Outside the cathode an anode 2 is located, which has a ring shape. The cathode is fastened on the anode 2 by the cylindrical sleeve 3 and the insulator 4 . In the inner cavity of cryostat 9 is liquid nitrogen. Inside of the cryostat 9 is a superconductor magnetic system consisting of three windings. The first winding 7 serves to create a tangential magnetic field of excitement in the working chamber. The second cylinder-shaped winding 14 serves to create a magnetic nozzle that compresses plasma at the exit of the working chamber. The third winding 19 is also cylindrical and is designed to stabilize the electric arc between electrodes 1 and 2 by means of its uniform rotation. Figure 14 shows that the coil of the first winding consists of two parts 3 and 4 . The part of the coil 3 with half-turns being reeled in a straight direction, is laid on the outer surface of the cylinder 6 . The other part of coil 4 with half-turns being reeled in the opposite direction, is being bent back and is stacked along radius. Similarly, the other three coils of the first winding are being laid. The fastening of the coils of the first winding is carried out with the help of a cylindrical bandage 13 and a flat bandage 10 .

Figure 13 shows cryostat 9 , which is inserted inside the outer cylinder 13. The magnetic system of windings is suspended with the help of disks 11 and 12 to the 


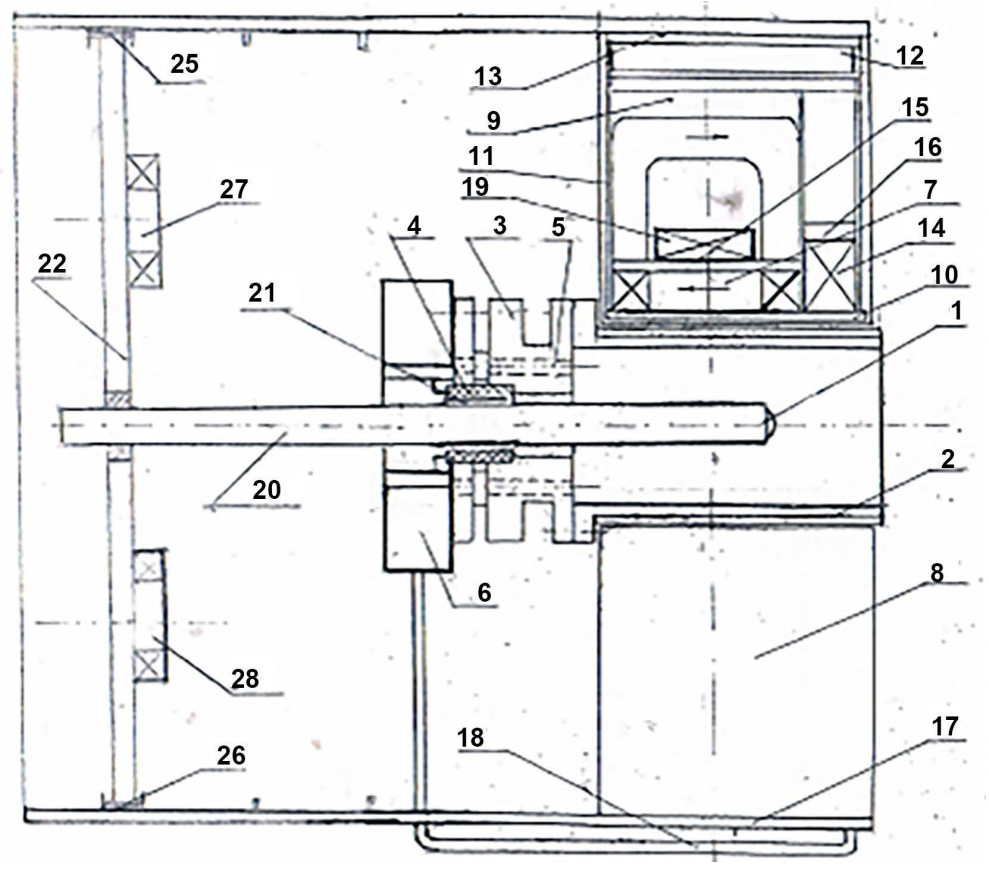

Figure 13. Longitudinal section of electrorocket engine MARS.

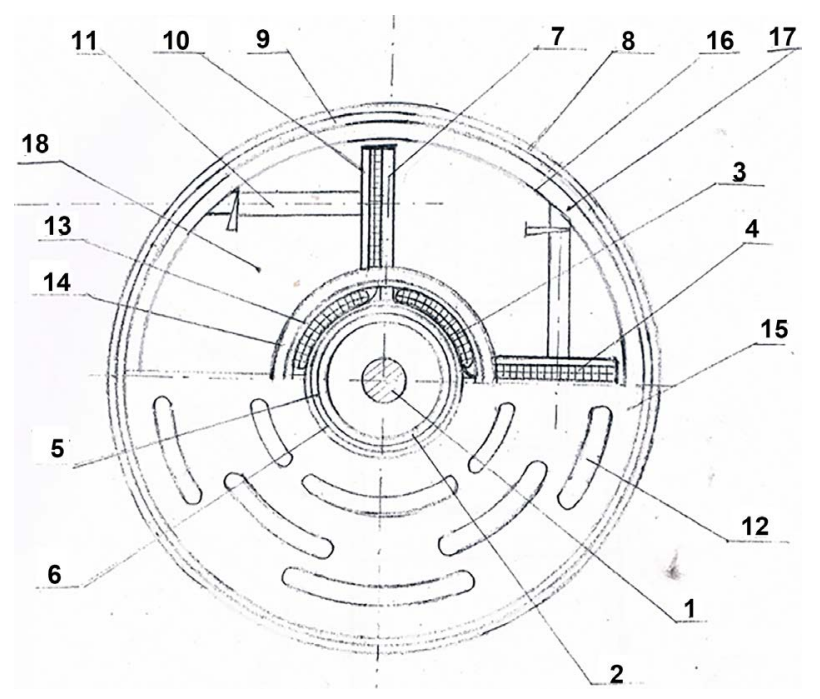

Figure 14. Cross section of electrorocket engine MARS.

cylinder13. Gaseous nitrogen, which is being formed during operation in the cryostat 9 , is transmitted through pipeline 18 to the chamber 6 of work substance preparation. Inside camera 6 is a dispenser and an electromagnetic valve. Gaseous nitrogen enters through the channel 5 in the working chamber of the electric motor.

When creating an engine of magnetoplasma-type with electrodes, there is the problem of resource. When the cathode is bombarded with high-energy ions, it loses mass. To solve this problem, the author developed a new electric motor design with a movable cathode [15].

During operation the cathode 1 (Figure 13) made of tungsten and having cy- 
lindrical shape moves along the horizontal axis of the engine. The end of the cathode 20 is rigidly fixed on the axis in the centre of the cross 22 , which is moving with the help of runners 25 and 26 along the inner surface of the cylinder 17.

In order to move the cathode 20 the magnetic force is used, to this purpose, the magnets 27 and 28 are installed in spokes 22 .

During operation, the electromagnets are in a constant magnetic field, which is created by the superconducting exciting winding 7 .

When electromagnets 27 and 28 are turned on, the cathode 20 due to the attractive force begins to move along the engine axis. The switching on of the electromagnets 27 and 28 occurs after a long operation of the engine, when current of the engine is reduced up to an unacceptable value due to the loss of cathode mass.

The calculated parameters and sizes of the electric motor MARS are shown in Table 1.

Table 1. The calculated parameters and sizes of the electric motor MARS.

\begin{tabular}{cc}
\hline Tractive force & $60 \mathrm{~N}$ \\
\hline Power & $600 \mathrm{~kW}$ \\
Current & $600 \mathrm{~A}$ \\
Voltage & $1000 \mathrm{~V}$ \\
Efficiency & $94 \%$ \\
Specific pulse & $6000 \mathrm{sec}$ \\
Working substance consumption & $1 \mathrm{~g} / \mathrm{sec}$ \\
Working substance discharge rate & $10 \mathrm{~km} / \mathrm{sec}$ \\
Magnetic inductance & $1,5 \mathrm{~T}$ \\
Anode diameter & $170 \mathrm{~mm}$ \\
Cathode diameter & $40 \mathrm{~mm}$ \\
Anode length & $100 \mathrm{~mm}$ \\
Cathode length & $60 \mathrm{~mm}$ \\
Diameter of the external cylinder & $600 \mathrm{~mm}$ \\
The length of the external cylinder & $450 \mathrm{~mm}$. \\
\hline
\end{tabular}

\section{Takeoff-Landing Capsule (TLC-1) of the Marsoplane}

The capsule is designed to move two astronauts from the north pole of Mars to any point on its surface.

The design of the capsule is a further modification of the design, which was developed by the author in 2016 for landing and taking off from the surface of the Uranus satellite-Oberon [16].

The design of TLC-1 is shown in Figure 15.

\section{Takeoff-Landing Capsule (TLC-2) for Interorbital Flight}

It is designed to move three astronauts from Earth orbit to Mars orbit, landing on the surface of Mars, take off into its orbit and flight from Mars orbit to Earth orbit. 


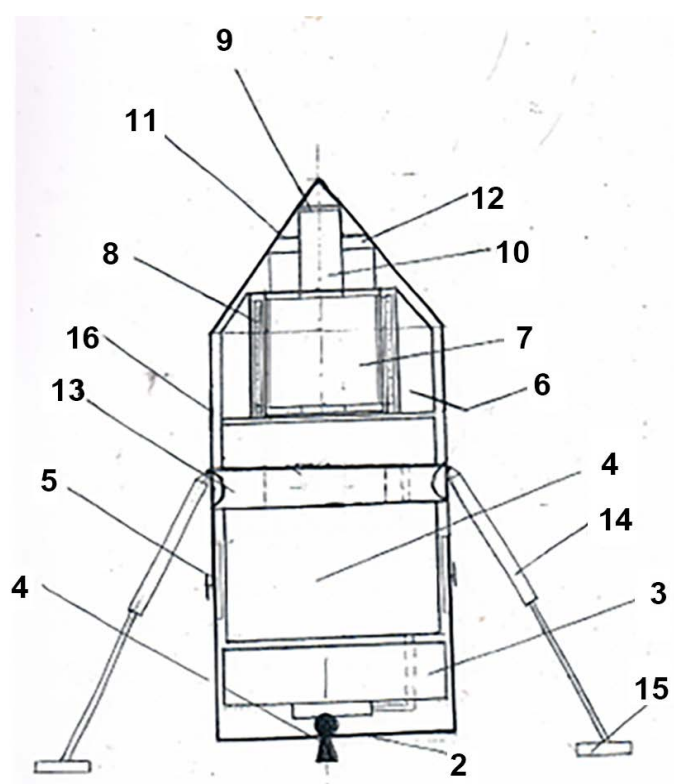

Figure 15. Takeoff-landing capsule TLC-1. where: 1) chemical hydrogen-oxygen rocket engine with tractive force of 20 tons and specific pulse of $400 \mathrm{sec}$; 2) bottom of rocket case of the capsule; 3) fuel tank with liquid oxygen; 4) fuel tank with liquid hydrogen; 5) chemical rocket engine for a manoeuvre; 6) tank with liquid hydrogen; 7) cabin for the expedition crew; 8) superconducting solenoid, designed to create a permanent magnetic field in the cabin; 9) docking unit for connecting with the ER-7 rocket; 10) a tunnel and a gateway for exit out of the rocket; 11) parachute system for landing on the surface of Mars; 12) container for measuring devices; 13) load bearing ring of the landing tripod; 14) damper of landing upright; 15) supporting foot of landing upright; 16) outer case of the TLC; TLC mass (on Earth) -22 t. TLC-1 length-15 m. TLC-1 external diameter-5, $8 \mathrm{~m}$.

The design of the TLC-2 is shown in Figure 16.

The design of the TLC- 2 is based on the spacecraft TLC- 1 with the preservation of the sizes of the capsule and has positions similar to those indicated in Figure 15.

The difference between TLC- 2 and TLC- 1 is the different size of tanks with working substance for chemical rocket engine 1 . It shall be recalled that after refuelling the TLC-1 must carry out two takeoffs and two landings on the surface of Mars, and TLC-2-one landing and one takeoff. Therefore, tank 4 with liquid hydrogen at TLC-2 has smaller volume than the TLC-1. Due to this the space for astronauts of cabin 7 at TLC-2. significantly increases.

Another constructive difference between TLC-1 and TLC-2 is due to the fact that during the orbital flight of TLC-2 the system of artificial gravity creation in the cabin of astronauts is activated, by means of the rotation of the capsule around the transversal axis. For this purpose, the docking unit 17 (Figure 16) is installed on the load bearing ring of the landing tripod 13 (on the transversal axis of the capsule). 


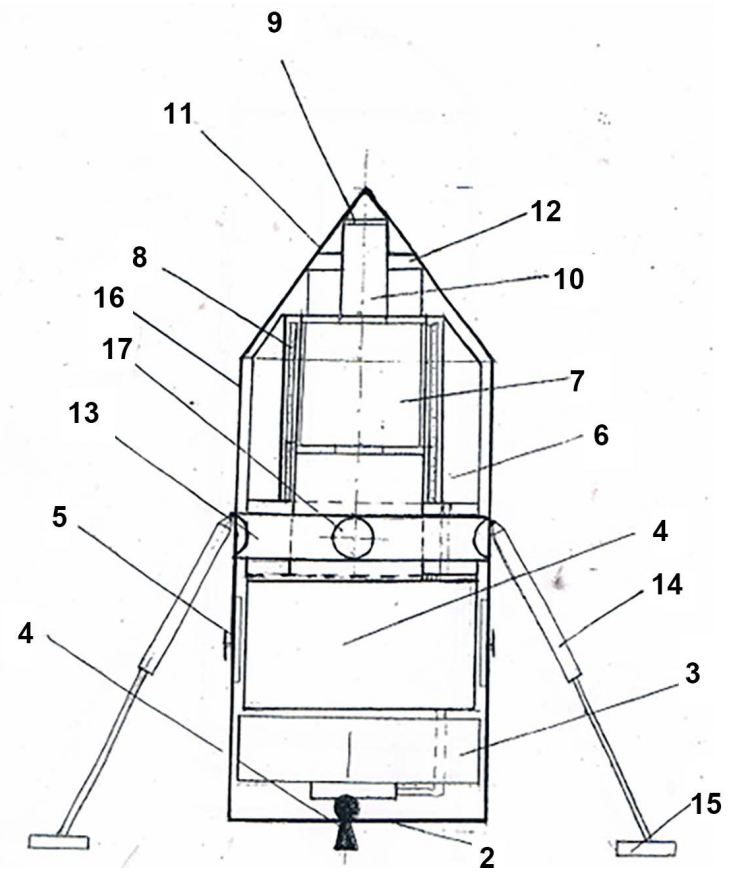

Figure 16. Takeoff-landing capsule TLC-2.

When the space train, shown in Figure 2 is formed, the TLC- 2 with the help of docking unit 17 is being connected with the docking unit 8 of rocket ER-7. At the end part of rocket ER-7 case is a superconducting bearing unit, in which the takeoff-landing capsule of the TLC-2 is rotated.

\section{Mobile Solar Panel}

It is designed to provide the expedition with electricity during the stay at the north pole of Mars. The mobile solar panel (MSP) is delivered at the north pole of Mars during a preliminary flight in accordance with scheme, shown in Figure 1. For landing on the icy surface of Mars the MSP has a chemical rocket engine.

The design of the MSP is shown in Figure 17.

The solar battery is a photovoltaic energy converter made using technology developed by Sharp. The basis is a three-cascade solar cell based on gallium arsenide with silicon substrate.

After landing on the surface of Mars, the astronauts remove the outer cone of the rocket 12 and using an attachment unload the assemblies 8 and 9 to the surface.

Astronauts install solar battery $\mathrm{N} 1$ from assembly 8 and solar battery $\mathrm{N} 2$ from assembly 9. The size of the solar panel in the working position: length $-100 \mathrm{~m}$, width $-5 \mathrm{~m}$. The power of each solar panel on the surface of the north pole of Mars- $180 \mathrm{~kW}$. The mass of solar panel on Earth is 4.2 tons.

\section{Space Refueller at the North Pole of Mars}

The refueller is an autonomous spacecraft that is designed to refuel takeoff- 


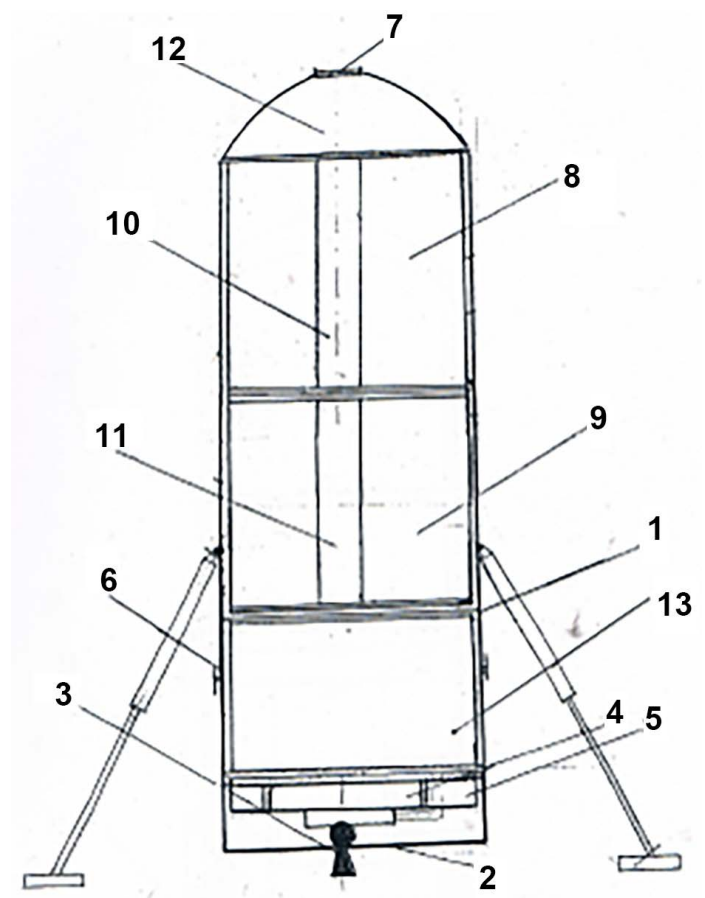

Figure 17. Mobile solar panel. where: 1) rocket body made of aluminium alloy; 2) rocket bottom; 3) chemical hydrogen-oxygen rocket engine with tractive force of $10 \mathrm{KN}$ and specific pulse of 400 seconds; 4) internal cylindrical tank with liquid hydrogen; 5) external cylindrical tank with liquid oxygen; 6) rocket engines for taxiing when docking with rocket; 7) docking unit for connection with the rocket ER-7; 8) upper assembly of solar panel; 9) lower assembly of solar panel; 13) cargo section for Mars rover and residential complex "Iglus".

landing capsules of TLC-1 and TLC-2, which are located on the surface near the north pole of the planet Mars.

The refueller melts the ice on the surface of Mars, performs electrolytic decomposition of water into hydrogen and oxygen, and then converts the components into liquid state. The liquid hydrogen and oxygen are pumped with the help of cryogenic pumps into tanks of chemical rocket engines intended for taking off of the TLCs from the surface of Mars.

The design of the refueller for the expedition to the north pole of Mars is shown in Figure 18.

\section{Conclusion}

1) Comprehensive scientific research and technical design of the expedition to the north pole of Mars has been carried out.

2) A method of two-stage delivery of astronauts to the icy surface of the north pole of Mars using an electric ER-7 rocket has been developed.

3) A technology of refuelling the chemical engine of the takeoff-landing capsule on the surface of the north pole of Mars with the help of a space refueller has been developed. 


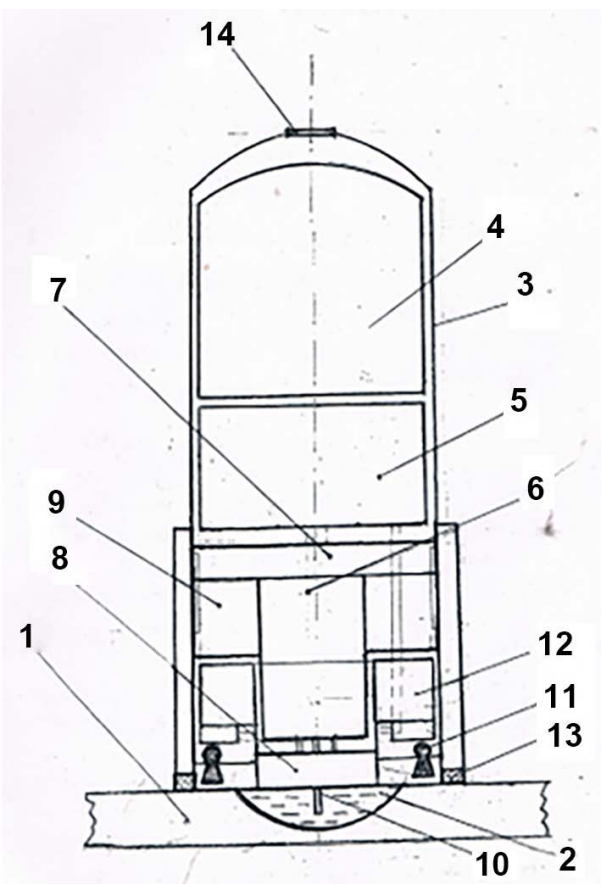

Figure 18. Space refueller. where: 1) Mars surface out of water ice at temperature $184^{\circ} \mathrm{K} ; 2$ ) unfrozen patch of wate, formed as a result of the thermal electric heater action; 3 ) external case of the rocket made of aluminium alloy; 4) a cylindrical hollow tank with liquid hydrogen, which is used as fuel for chemical rocket engines; 5) cylindrical hollow tank with liquid oxygen, which is used as oxidizer in chemical rocket engines; 6) electrolyser for the production of hydrogen and oxygen from liquid water. The power of the electrolyser is $85 \mathrm{~kW}$; 7) liquefier for converting gaseous hydrogen and oxygen into liquid state; 8) thermoheater. The thermoheater has cylindrical shape and is installed at the bottom of the rocket on its longitudinal axis, on which it moves with the help of a pneumatic motor. Constructively the thermoheater consists of separate blocks of nichrome electro heaters, which are connected sequentially Power of the electro heater. $120 \mathrm{~kW}$; 9) tank with liquid oxygen on liquefier 7 exit; 10) pipeline for water feeding from unfrozen patch of water into electrolyser; 11) chemical rocket engine for landing on the surface of Mars. Rocket engine thrust-12 KN; 12) fuel tank for chemical rocket engine 11 ; 13) sleigh skid to move the refueller on the icy surface of the Mars north pole; 14) docking unit for connection with ER-7 rocket that delivers the refueller into orbit around Mars.

4) The design of the spacecraft-a marsoplane, which with the help of an electric rocket ER-7 and take-off-landing capsule TLC-1 allows to fly from the north pole to any point on the surface of Mars has been developed.

5) The project envisages the creation of a permanent research base at the north pole of Mars, which will carry out complex measurements of the climatic and physical parameters of Mars.

6) As a result of the calculation, it is shown that the set of proposed new design solutions will allow to carry out regular flights to the north pole of Mars 
every two years and the duration of the flight from Earth orbit to the orbit of Mars will be 40 days.

\section{Conflicts of Interest}

The author declares no conflicts of interest regarding the publication of this paper.

\section{References}

[1] Eugen Reichl (2012) Zukunftsprojekte der Raumfahrt. Motor Verlag.

[2] Bischoff, J. (2015) Mission Mars. GEO N 11, S.51-64.

[3] Troutman, P. (2015) Reise zum Mars Space 5/S 24-27.

[4] Onken, J. (2016) Die grösste Rakete der Welt Space 3/S 62-69.

[5] Rubinraut, A. (2017) The Study on Electrorocket Engine for the Future AAST, 2, 14, 6.

[6] Rubinraut, A. (2018) The Space Cruise over the Route Earth-Mars-Earth. AAST, 3, 20-47. https://doi.org/10.4236/aast.2018.32003

[7] Smith, P.H., et al. (2009) $\mathrm{H}_{2} \mathrm{O}$ at the Phoenix Landing Site. Science, 325, 58-61. https://doi.org/10.1126/science.1172768

[8] Rubinraut, A. (2017) The Expedition to Jupiter. IETAE, Volume 2 Number 1 January.

[9] Рой (1991) Движение по орбитам. Мир. Москва. (In Russian)

[10] Iglus auf dem Mars. Space 4. 2017 S. 80.

[11] Unglaubliche Weltraum Raboter. Space 2 2015. S. 3.

[12] MARS-ROVER Space 4. 2017 S.71.

[13] Krisciunas, K. and Yenne, B. (1998) Atlas des Universums, Lechner.

[14] Rubinraut, A. (2013) Elektrischer Düsenantrieb für dem Flug zum Mars. Patent Nr.102006022 559 DPMA.

[15] Rubinraut, A. (2016) Elektrisches Raketentriebwerk für Dauerweltraumfllüge. Patent Nr 2013 08, 2 DPMA München.

[16] Rubinraut, A. (2016) The Expedition towards Uranium. IETAE Volume 6 Issue 7 July. 